\title{
Update on the efficacy, effectiveness and safety of artemether-lumefantrine combination therapy for treatment of uncomplicated malaria
}

This article was published in the following Dove Press journal:

Therapeutics and Clinical Risk Management

16 December 2009

Number of times this article has been viewed

\author{
Pauline Byakika-Kibwika ${ }^{1,2}$ \\ Mohammed Lamorde ${ }^{1,2}$ \\ Harriet Mayanja-Kizza' \\ Concepta Merry ${ }^{1,2}$ \\ Bob Colebunders ${ }^{3,4}$ \\ Jean-Pierre Van geertruyden ${ }^{4,5}$
}

'Infectious Diseases Institute and Infectious Diseases Network for Treatment and Research in Africa (INTERACT), Faculty of Medicine, Makerere University, Kampala Uganda; ${ }^{2}$ Trinity College Dublin, Ireland; ${ }^{3}$ Dienst Clinical Sciences, Instituut voor Tropische Geneeskunde, Antwerp, Belgium; ${ }^{4}$ International Health, Epidemiology en Social Medicine, Universiteit Antwerpen, Antwerp, Belgium; ${ }^{5}$ Epidemiology Unit, Dienst Parasitologie, Instituut voor Tropische Geneeskunde, Antwerp, Belgium
Correspondence: Jean-Pierre Van geertruyden

Unit International Health,

Department of Epidemiology and

Social Medicine, Antwerp University,

Universiteitsplein, I, BE-26I0

Antwerpen, Belgium

Tel +3232652528

Fax +32484618493

Email jean-pierre.vangeertruyden@ua.ac.be

\begin{abstract}
Artemether-lumefantrine is one of the artemisisnin-based combination therapies recommended for treatment of uncomplicated falciparum malaria. The drug combination is highly efficacious against sensitive and multidrug resistant falciparum malaria. It offers the advantage of rapid clearance of parasites by artemether and the slower elimination of residual parasites by lumefantrine. The combination can be used in all populations except pregnant mothers in the first trimester where safety is still uncertain. There are still concerns about safety and pharmacokinetics of the drug combination in children, especially infants, pregnant mothers and drug interactions with mainly non-nucleoside reverse transcriptase inhibitors and protease inhibitors used for HIV therapy.
\end{abstract}

Keywords: artemether-lumefantrine, efficacy, effectiveness, safety, malaria

\section{Introduction}

Malaria is a febrile illness caused by intracellular protozoa of the genus Plasmodium, and transmitted by the bite of an infected female mosquito of the genus Anopheles. Plasmodium species that cause disease in humans include: P. falciparum, P. vivax, P. malariae, $P$. ovale, and P. knowlesi. P. falciparum is the most prevalent and most virulent. Worldwide, malaria is one of the most important causes of morbidity and mortality. Approximately 2.2 billion people are exposed to malaria every year of whom about 300 to 500 million develop disease. In 2006, there were 247 million cases of malaria, causing nearly 1 million deaths, mostly among African children. ${ }^{1}$ Malaria deaths are responsible for almost $3 \%$ of the world's disability-adjusted life years, not counting the considerable and imprecisely quantified burden due to morbidity and disability. ${ }^{2}$ In addition to causing significant morbidity and mortality, malaria significantly contributes to poverty through lost productivity and economic loss on antimalarial treatment. African countries spend US\$12 billion annually on malaria, with individual African families spending up to $25 \%$ of their income on malaria prevention and control. Malaria has slowed economic growth in African countries by $1.3 \%$ per year. As a result of the compounded effect over 35 years, the gross domestic product for African countries is now up to $32 \%$ lower than it would have been in absence of malaria. ${ }^{3}$

Reduction in malaria-associated morbidity and mortality largely depends on provision of prompt, effective, safe and affordable antimalarial drugs. Resistance to antimalarial drugs poses a significant challenge to malaria control programs in sub-Saharan Africa. Multi-drug resistance to sulfadoxine-pyrimethamine (SP) and chloroquine was described extensively in sub-Saharan Africa. The World Health 
Organization (WHO) recommends use of artemisinin-based combination treatments (ACT) as first-line therapy. The ACTs combine fast-acting artemisinins with another structurally unrelated and more slowly eliminated compound which permits elimination of residual malarial parasites. ${ }^{4-6}$ Of the 81 countries with endemic $P$. falciparum, 77 have now adopted the WHO recommendation. ${ }^{7}$ Commonly used ACTs are artemether-lumefantrine (AL), amodiaquine-artesunate (AQAS), mefloquine-artesunate, dihydroartemisinpiperaquine (DP) and napthoquine-artemisinin. In this review we provide an update on efficacy, effectiveness and safety of AL for treatment of uncomplicated malaria.

\section{Pharmacology of artemether- lumefantrine}

A 6-dose regimen of artemether $(20 \mathrm{mg})$ co-formulated with lumefantrine $(120 \mathrm{mg})$ is recommended; with first and second doses taken 8 hours apart, the third dose taken 24 hours after the first and the remaining doses 12 hours apart. The 6-dose regimen is superior to the 4-dose regimen. ${ }^{8,9}$ Artemesinin from which artemether is derived is obtained from the Chinese herb sweet wormwood (Artemisua annua). Artemisinins have the most potent and rapid onset of antiparasitic activity against all Plasmodium species that infect humans.

Artemether acts rapidly with half-life of 1 to 3 hours, whereas lumefantrine has a half-life of 3 to 6 days and is responsible for preventing recurrent parasitemia. ${ }^{10}$ Artemether and lumefantrine have different modes of action and act at different points in the parasite life cycle. ${ }^{11,12}$ Artemether interferes with parasite transport proteins, disrupts parasite mitochondrial function, inhibits angiogenesis and modulates host immune function. ${ }^{13}$ Lumefantrine is an aryl-amino alcohol ${ }^{14}$ that prevents detoxification of heme, such that toxic heme and free radicals induce parasite death. ${ }^{12}$ Oral formulations of AL are available as tablet and dispersible formulations which have similar pharmacokinetic (PK) properties. ${ }^{15,16}$ Artemether and lumefantrine differ in rates of absorption and elimination. Artemether is rapidly absorbed reaching peak plasma concentrations within 2 hours post dose. ${ }^{11,17}$ It is metabolized rapidly by cytochrome P450 (CYP) 2B6, CYP3A4 and possibly CYP2A610 to dihydroartemisinin (DHA) which in turn is converted to inactive metabolites primarily by glucoronidation via UGT1A1, 1A8/9 and 2B7. ${ }^{14}$ The metabolite DHA reaches peak plasma concentration within 2 to 3 hours post dosing. ${ }^{11}$ Both artemether and DHA offer potent antimalarial properties causing significant reduction in asexual parasite mass of approximately 10,000-fold per reproductive cycle, with prompt resolution of symptoms. ${ }^{18,19}$
Lumefantrine is absorbed and cleared more slowly, acting to eliminate residual parasites that may remain after artemether and DHA have been cleared from the body and thus prevent recrudescence. ${ }^{11,12}$ Lumefantrine is highly lipophilic, thus absorption is enhanced with a fatty meal; its absorption occurs 2 hours after intake reaching peak plasma concentration after 3 to 4 hours ${ }^{20}$ with an elimination half life of 4 to 10 days. $^{20,21}$

Food enhances absorption of both artemether and lumefantrine although this effect is more apparent for lumefantrine. ${ }^{11,20}$ Administration of AL with high-fat meal increased bioavailability of both artemether and lumefantrine by 2-fold and 16-fold respectively. ${ }^{11}$ Premji et al in an evaluation of the typical fat content of African diets noted that total fat intake is 15 to $30 \mathrm{~g} /$ day during breast feeding, $>10 \mathrm{~g} /$ day in the post weaning phase and 30 to $60 \mathrm{~g} /$ day in a normal diet and this is adequate for optimal efficacy of lumefantrine. ${ }^{22}$

However, the effect of food on AL absorption is of concern because patients with malaria usually have anorexia, vomiting and low food intake. Lumefantrine is metabolized by N-debutylation mainly by CYP3A410 to desbutyllumefantrine with 5- to 8-fold higher antiparastic effect than lumefantrine. The key PK determinant of cure is the area under the concentration time curve (AUC) of the longeracting lumefantrine.

\section{Efficacy and effectiveness of AL}

Efficacy of the 6 dose regimen of AL judged by elimination of malaria parasites using the 28-day polymerase chain reaction (PCR)-corrected cure rates and resolution of symptoms, has been demonstrated in semi-immune and non-immune populations in Asia and Africa to be consistently greater than $95 \%$, with rapid parasite and symptom clearance and significant gametocidal effect. ${ }^{1523-27}$ Many studies in Africa and Asia have demonstrated AL to be as efficacious as other ACTs when used in pediatric and adult populations with differing immunity. PCR-corrected day 28 and day 42 cure rates range between $91 \%$ and $100 \%$ using evaluable patient analysis. ${ }^{28-62}$ Correction by PCR enables differentiation between recurrence and recrudescence of the initial infection from re-infection. A few cases of treatment failure were recorded after AL treatment, but these were mostly re-infections..$^{29,32,33,38}$ This is of particular concern in areas with very intense malaria transmission where antimalarial drugs with longer half-life may offer the advantage of preventing re-infection. Lumefantrine with an estimated elimination half-life of 4 to 10 days offers post-treatment antimalarial prophylaxis of up to 4 weeks. Studies showed 
both AL and DP to be highly efficacious for treatment of uncomplicated malaria, although DP was superior to $\mathrm{AL}$ at preventing new malaria infections..$^{33,34,38,50}$ In addition to excellent efficacy and effectiveness, AL has demonstrated significant gametocidal effects. ${ }^{34,42,48,51}$ A meta-analysis of 32 randomized trials showed AL to be one of the most effective ACTs with 28-day parasitological cure rates of 97.4\% ${ }^{63}$

Effectiveness of AL may be influenced by poor adherence to the 3-day, 6-dose regimen and the food requirements for $\mathrm{AL}$ absorption. Clinical and parasitological responses to AL were similar with both supervised and unsupervised treatment in Uganda. ${ }^{64}$ The supervised treatment arm received AL with fatty food while the unsupervised arm received AL as outpatient treatment with nutritional advice. Unsupervised treatment resulted in lower concentrations of lumefantrine with increased risk of early reinfection., ${ }^{4,64}$ In Uganda and Nigeria adherence to correct AL dose and duration prescribed to febrile children by community medicine distributors was greater than $80 \%$ and crude parasitological failure rates varied from $3.7 \%$ in Uganda to $41.8 \%$ in Nigeria and PCR-adjusted parasitological cure rate was $90.9 \%$ in Nigeria and $97.2 \%$ in Uganda. ${ }^{6}$ Differences in crude rates may be due to differences in re-infection rates. A recent study of uncomplicated malaria in Uganda showed adherence to AL was $94.5 \%$ compared to that quinine of $85.4 \%$ with high unadjusted cure rates of AL of $96 \%$ vs $64 \%$ for quinine. ${ }^{65}$

In multidrug-resistant areas, day 7 lumefantrine concentration was a useful surrogate marker for AUC and concentrations of less than $280 \mathrm{ng} / \mathrm{mL}$ predicted treatment failure. ${ }^{17,20}$ However, results from areas with lumefantrinesensitive parasites showed no treatment failures despite day 7 concentrations less than $280 \mathrm{ng} / \mathrm{mL}$ in $45 \%$ of all patients, and re-infections occurred among patients with day 7 concentrations below $400 \mathrm{ng} / \mathrm{mL}$ and those who received a lower dose of lumefantrine per kilogram body weight. ${ }^{4}$

\section{Safety of AL}

Safety and tolerability of AL has been assessed in clinical trials in Asia and Africa. Most adverse events are mild or moderate, mostly affecting gastrointestinal and nervous systems; however, most are typical of the symptomatology of malaria or concomitant infections. ${ }^{15,24-27,66}$ Serious adverse events were unlikely and were unrelated or most unlikely to be related to study medication. ${ }^{15,29-31,33,34,36,38,39,41-43,46-51,53,54,67}$ Two meta-analysis concluded that AL is well tolerated, with mild or moderate adverse events mostly affecting gastrointestinal and nervous systems. Ototoxicity associated with AL has been reported recently in a few cases $;{ }^{68,69}$ however, this was not confirmed in a study that investigated hearing sensation following AL treatment. ${ }^{31}$ Lumefantrine possesses a similar chemical structure to halofantrine which is known to cause cardiac arrhythmia; however, safety studies have not shown lumefantrine to be cardiotoxic or to prolong QTc interval. ${ }^{67,70}$ Other studies and a review of 15 trials concluded that AL did not cause hematological adverse events, although pre-clinical trials suggested the repeated exposure to AL may affect blood cell counts. $^{71}$

Safety assessment has been conducted during treatment of single episodes of malaria. Safety concerns become more important when AL is administered over the counter, which commonly results in overdiagnosis and overtreatment of malaria, and when patients get recurrent infections requiring repeated treatment. Overdiagnosis of malaria is common in malaria-endemic areas. ${ }^{72}$ There are no standard guidelines for evaluating drug safety and tolerability in antimalarial trials. ${ }^{64}$ Establishing systems for pharmacovigilance in areas where AL is frequently prescribed is of utmost importance and several challenges exist. ${ }^{73}$

\section{AL use in children}

Vomiting, which may be due to disease-related nausea or taste of the medication, may influence drug intake especially in children. A more palatable dispersible formulation of AL is now available and has been shown to be as efficacious as the currently used crushed tablet in infants and children, and with similar safety and PK profile. ${ }^{15}$ Pediatric dosing of AL is deduced from adult-based regimens adjusted for body weight, with little consideration for maturational effects on drug absorption and metabolism. Although diet and nutritional status are important determinants of PK processes, drug responses and toxicity, there are few relevant data for AL in this patient group. In resource-constrained areas, children may not be weighed at each clinic visit and dosing in such settings is usually based on age as a proxy measure for weight. Besides research on therapeutic dose levels based on body weight, there is urgent need for evidence-based translation of weight based dosing regimens to regimens that can be based on age, as the majority of fevers in malaria endemic areas are treated with over-the-counter antimalarial drugs without involvement of the formal health sector. Age-based dose regimens are more practical than weight-based regimens, but will inevitably result in a greater proportion of children receiving either too much or too little drug. This is a particular concern with lumefantrine, which has a narrow therapeutic margin between effective and toxic concentrations. This dosing consideration is especially important in malnourished, 
pre-school children and during onset of puberty when physiological variations in bodyweight by age are greatest. Earlier experience with SP and DP suggests that lack of clear guidance on age-based dosing as part of the regulatory process contributes to considerable variation in recommended age-based dose regimens, ${ }^{74,75}$ potentially resulting in poor, but widely used regimens, particularly for young rapidly growing children who bear the brunt of the malaria burden. Different age-based regimens are already being used in countries that have recently switched to ACTs. These concerns apply also to young infants $<6$ months old or of $<5 \mathrm{~kg}$ body weight. Most ACTs are contra-indicated in this group because of lack of safety data, even though these children are at considerable risk. In western Kenya $50 \%$ of infants not protected by insecticide-treated mosquito nets had their first infection by 3 months. ${ }^{76}$ In southern Mozambique, an estimated 9\% of out-patient visits for uncomplicated malaria in children less than 5 years of age are children aged $<6$ months. Infants in endemic areas have the highest burden of severe malarial anemia, blood transfusions and death. ${ }^{77,78}$ Thus, programmatically implemented ACTs will end up being widely used in children $<6$ months even though the label does not provide guidance for this age group.

\section{Malaria and AL use in pregnancy}

Pregnant women with malaria, symptomatic and asymptomatic alike, should be treated without delay with effective and safe antimalarial drugs in order to reduce risks for adverse outcomes for both mother and fetus. ${ }^{79} \mathrm{AL}$ is a very attractive alternative because it is highly effective, acts rapidly and is well tolerated. However, there is insufficient information on safety and efficacy of ACTs in pregnancy, including exposure in the first trimester. ${ }^{79,80}$ Early data indicated that artemisinins were embryotoxic and potentially teratogenic in several animal species without maternal toxic effects or impaired fertility, and more recent studies have confirmed these findings. ${ }^{79}$

Artemisinin derivatives have shown embryo-toxic effects in animal reproductive toxicology studies.$^{81}$ The mechanism of embryo-toxicity is thought to occur through depletion of embryonic erythroblasts causing severe anemia and cell damage and death due to hypoxia. ${ }^{81}$ The most sensitive time window for embryo-toxicity in humans is between weeks 4 to 10. From these data ACTs are not indicated for malaria treatment in the first trimester of pregnancy unless no alternatives exist. There is increasing experience with artemisinin derivatives in second and third trimesters with no evidence of adverse outcomes in more than 1000 prospectively followed pregnancies. ${ }^{82,83}$ WHO Malaria Treatment Guidelines of 2006 recommend use of ACTs in pregnant women in the second and third trimester of gestation. None of the studies on AL use in pregnancy have reported increased risk of serious maternal adverse events, adverse birth outcomes or neuro-developmental deficits. However all these studies were underpowered to detect rare adverse outcomes. ${ }^{84}$ Data from Sudan from a cohort of women who reported use of artemisinins in first trimester and were followed up until delivery and their babies followed up till 1 year of age showed that most delivered apparently healthy babies at full term with no congenital malformations and no maternal deaths, and none of the babies died during their first year of life. ${ }^{85} \mathrm{~A}$ prospective observational study was conducted recently in Zambia which evaluated safety of AL and SP in pregnant women who received $\mathrm{AL}$ and SP to treat symptomatic falciparum malaria. Data from 1001 pregnant women and fetuses/newborns indicated that the incidence of perinatal death, spontaneous abortion, neonatal mortality, premature delivery, stillbirth and low birth weight is similar after pregnancy exposure to AL compared to SP. ${ }^{86}$

Pregnancy has been associated with reduced plasma concentrations of AL which have a significant impact on treatment outcome since plasma concentrations of lumefantrine, after elimination of artemether, are an important determinant of cure. ${ }^{87,88} \mathrm{~A}$ study that evaluated PK of $\mathrm{AL}$ in pregnant women with recrudescent uncomplicated multidrug resistant falciparum malaria demonstrated that pregnant women in second and third trimester had lower concentrations of artemether, dihydroartemisinin and lumefantrine, and elimination of lumefantrine was more rapid than reported previously in non-pregnant adults. ${ }^{87,89}$ Another study that compared artesunate monotherapy to AL for treatment of uncomplicated falciparum malaria in second and third trimesters demonstrated that the standard 6-dose AL regimen was well tolerated and safe but efficacy was inferior to that of 7-day artesunate monotherapy and was unsatisfactory for general deployment in this geographic area. PK parameters measured in this study showed low drug concentrations in later pregnancy which could possibly explain the poor treatment outcomes ${ }^{89}$ There is need for further studies to determine the optimum dose regimen and efficacy of AL in pregnancy.

\section{AL use in HIV-infected populations}

Human immunodeficiency virus (HIV)-infected individuals are at high risk for acquiring malaria parasitemia, with the risk increasing as immunity declines. ${ }^{90-93}$ Evidence for this interaction is more consistent in pregnant women of all gravidities. ${ }^{94-96} \mathrm{HIV}-1$ infected pregnant women have a higher prevalence of peripheral parasitemia and placental malaria ${ }^{95,96}$ 
and their infants experience higher postnatal mortality when both diseases are present. ${ }^{97,98}$ Therefore, offering adequate and efficacious antimalarial treatment and prevention is extremely important for this high risk group. Little is known about efficacy and safety of antimalarial drugs in HIV-infected individuals and much less on interaction between antimalarial and antiretroviral (ARV) drugs, and reliable data are urgently needed. Few studies have examined the effect of HIV infection on response to antimalarial treatment and these have yielded conflicting results. ${ }^{99-103}$ Most studies have shown that HIV-infected individuals have higher risk of experiencing antimalarial treatment failure due to re-infections. ${ }^{101,103}$ Birku et al demonstrated decreased clearance of parasites by artemisinin treatment in HIV-infected patients with malaria. ${ }^{104}$ In Zambia, HIV-infected adult patients with CD4 counts of $300 / \mu \mathrm{L}$ and below had higher risk of getting recrudescent malaria than HIV-infected patients with higher CD4 counts and HIV-uninfected patients. ${ }^{103}$ Recent studies, however, suggest that the threshold for an increased risk of malaria treatment failure (new infections or recrudescence) probably lies at $400 \mathrm{CD} 4$ cells/ $\mu \mathrm{L} .{ }^{105,106}$ Following the latest WHO guidelines for sub-Saharan Africa this malaria vulnerable population should be protected by cotrimoxazole prophylaxis or highly active ARV therapy (HAART). There are concerns about safety of AL treatment in HIV-infected patients concomitantly receiving HAART. The standard first-line HAART regimens in many sub-Saharan countries where malaria is endemic are made up of a non-nucleoside reverse transcriptase inhibitor (NNRTI) backbone with 2 nucleoside reverse transcriptase inhibitors (NRTI). The second-line HAART regimen is made up of a protease inhibitor (PI) backbone and 2 NRTIs. Knowledge of the metabolism of ARVs and AL suggests that there is potential for PK drug-drug interactions. ${ }^{107}$ For example, PIs like lopinavir/ritonavir (LPV/r) are among the most potent inhibitors of cytochrome P450 (typically CYP 3A4) metabolism, while NNRTIs (efavirenz and nevirapine) are also substrates of cytochrome P450 and usually these two induce but occasionally efavirenz inhibits some P450 isoforms. Although poorly studied the risk of clinically significant interactions involving AL and ARVs is considerable ${ }^{108}$ and may result in high concentrations with excessive toxicity or reduced concentrations with reduced efficacy and risk for development of resistance to AL. The potential for interactions between ARVs and antimalarials have been shown in a study of healthy volunteers where AQAS was co-administered with the NNRTI efavirenz. In the first 2 study participants, the AUC for AQAS increased by $100 \%$ to $300 \%$ and alanine and aspartate transferase levels increased markedly above the upper limit of normal, suggesting hepatoxicity. This led to recommendations that AQAS should be avoided in patients receiving EFV. In a recent study of uncomplicated malaria in Uganda, treatment of HIV-infected children with AQAS was associated with markedly higher risk of neutropenia compared with treatment of HIV-uninfected children. The risk of neutropenia was higher in participants with concurrent ARV use, especially zidovudine, and in those with a history of repeated doses of AQAS. ${ }^{109}$ These clinical observations demonstrate the need for thorough examination of the nature of interaction between ARVs and ACTs. An interaction is expected between lumefantrine and both EFV and PIs that could potentially lead to increased levels of lumefantrine (Figure 1); no data are available. The potential interactions with NVP are less clear but co-administration could reduce lumefantrine levels. A study that investigated the PKs of AL when administered with LPV/r in HIV-uninfected healthy volunteers demonstrated that the PK of lumefantrine is influenced by $\mathrm{LPV} / \mathrm{r}$, resulting in 2- to 3 -fold increases in lumefantrine $\mathrm{AUC}$, and trends towards decreases in artemether maximum concentration $\left(\mathrm{C}_{\max }\right)$ and $\mathrm{AUC}$ were noted during co-administration. Decreases in DHA AUC were observed during co-administration without changes in DHA: artemether AUC ratios. The authors concluded that co-administration of AL and LPV/r can be carried out for patients co-infected with malaria and HIV. ${ }^{10}$ This study did not address safety concerns with co-administration, which need to be considered in future studies among individuals living in malaria-endemic regions.

\section{AL use in patients with co-morbidity}

Treatment of tuberculosis is often a minimum of 6 months including 2 months of intense rifampicin-based treatment. Patients may concomitantly develop malaria requiring treatment with AL. There are currently no published data on interactions of rifampicin and AL. Rifampicin is a potent inducer of hepatic cytochrome and may influence the PKs of AL since both drugs are metabolized by CYP $450 .{ }^{111}$ Theoretically co-administration of rifampicin with AL may result in decreased concentrations of AL resulting in decreased efficacy (Figure 1). Data on these PK drug interactions are very scarce, thus the need for more studies. One study evaluated effects of concomitant administration of AL with a potent CYP 3A4 inhibitor. Artemether, DHA, and lumefantrine PKs were altered by ketoconazole. AUC and $\mathrm{C}_{\max }$ increased for all 3 compounds and terminal half-life increased for artemether and DHA. None of the changes in 


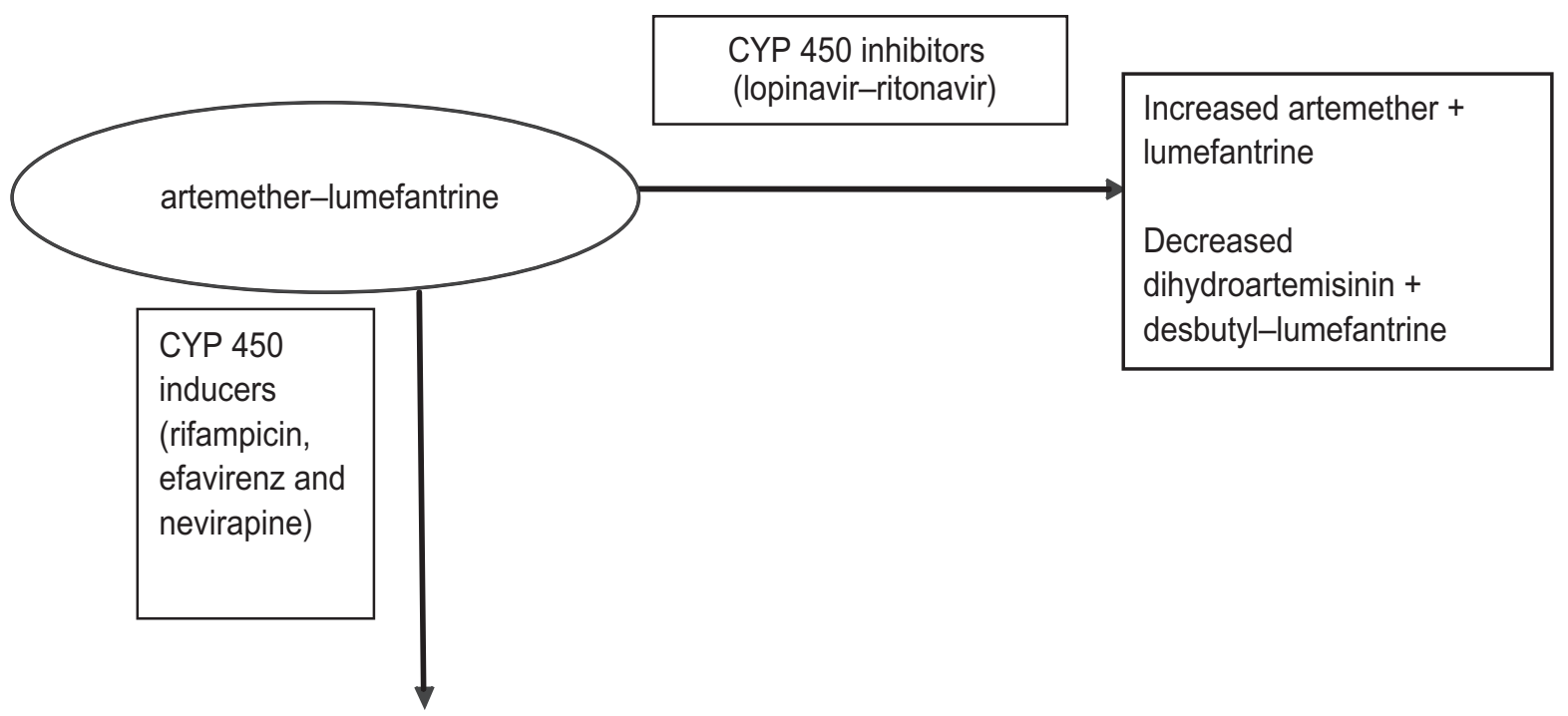

Decreased artemether + lumefantrine

dihydroartemisinin

Increased dihydroartemisinin + desbutyl-

lumefantrine

Figure I Summary of potential pharmacokinetic interactions between artemether-lumefantrine and commonly prescribed inducers and inhibitors of CYP 450.

PK parameters were greater than the changes observed in healthy volunteers taking AL with a high-fat meal. There was no increase in observed side effects or electrocardiographic changes. The authors concluded that dosage adjustments of AL do not appear to be necessary with concomitant ketoconazole administration. ${ }^{112}$

\section{$A L$ resistance}

Antimalarial drug resistance has been defined as "the ability of a parasite strain to survive and/or multiply despite the administration and absorption of a drug given in doses equal to or higher than those usually recommended, but within the limits of tolerance of the subject." This definition was later modified to specify that the drug in question must gain access to the parasite or the infected red blood cell for the duration of the time necessary for its normal action." ${ }^{113}$ Antimalarial drug resistance is heightened in individuals with lower immunity, such as children less than 5 years, pregnant women, non-immune immigrants to malarious areas, malnourished individuals and HIV-infected patients. ${ }^{113}$ Reduced immunity allows the survival of a residuum of parasites that are able to survive treatment, and as such reduced immunity may further increase the development, intensification and spread of resistant strains.

Resistance to artemisinins has not been confirmed although reduced sensitivity has been reported in China and Vietnam. ${ }^{14,115}$ Treatment failures occurring after $\mathrm{AL}$ treatment are thought to be due to poor absorption with reduced concentrations. ${ }^{116,117} \mathrm{AL}$ selects for the P. falciparum multidrug resistance gene (PfMDR1) N86, the chloroquinesusceptible allele which has been proposed as a marker for lumefantrine resistance. ${ }^{118}$ In Tanzania, treatment with $\mathrm{AL}$ was associated with selection of newly infecting parasites containing the $p f m d r 186 \mathrm{~N}$ allele, ${ }^{118}$ which has been associated with decreased in vitro sensitivity to artemisinins and lumefantrine. ${ }^{119}$

Factors that lead to development, intensification and distribution of antimalarial drug resistance can broadly be classified as: factors leading to treatment failure (incorrect dosing regimen, non-compliance, substandard drugs and misdiagnosis), human behavior, parasite and vector biology, and drug PKs. ${ }^{113}$ In sub-Saharan Africa antimalarial drugs are readily available outside public health services, in pharmacies, drug shops and private practitioners' clinics. 
Quality of antimalarials is a serious concern and counterfeits may be found in some of these units. In Southeast Asia half of the samples of artemisnins obtained from most countries were counterfeit. ${ }^{11,120}$ In sub-Saharan Africa substandard antimalarials were found in 7 countries. ${ }^{121,122}$

\section{Conclusion}

There is increasing evidence of very high efficacy and effectiveness of AL for treatment of uncomplicated malaria. Continued health education on correct use of AL and surveillance of effectiveness is necessary to prevent and detect emergence of drug resistance. There is need to develop strong systems for pharmacovigilance to increase the evidence base on safety of AL especially in pregnant mothers and infants weighing less then $5 \mathrm{~kg}$. PK studies especially on drug interactions with ARV drugs are urgently needed.

\section{Disclosures}

None of the authors declare conflicts of interest.

\section{References}

1. WHO. http://wwwwhoint/mediacentre/factsheets/fs094/en/indexhtml. Accessed July 2, 2009.

2. Breman JG, Alilio MS, Mills A. Conquering the intolerable burden of malaria: what's new, what's needed: a summary. Am J Trop Med Hyg. 2004;71(2):1-15.

3. WHO/CDS/RBM/2000.17. The Abuja Declaration and the Plan of Action. 2000 April 25.

4. Checchi F, Piola P, Fogg C, et al. Supervised versus unsupervised antimalarial treatment with six-dose artemether-lumefantrine: PK and dosage-related findings from a clinical trial in Uganda. Malar $J$. 2006;19(5):59.

5. Mutabingwa TK, Anthony D, Heller A, et al. Amodiaquine alone, amodiaquine + sulfadoxine-pyrimethamine, amodiaquine + artesunate, and artemether-lumefantrinefor outpatient treatment of malaria in Tanzanian children: a four arm randomised effectiveness trial. Lancet. 2005;365:1474-1480.

6. Ajayi IO, Browne EN, Bateganya F, et al. Effectiveness of artemisininbased combination therapy used in the context of home management of malaria: a report from three study sites in sub-Saharan Africa. Malar $J$. 2008;27(7): 190 .

7. WHO. World Malaria Report. Geneva: World Health Organization; 2008.

8. Omari AA, Gamble CL, Garner P. Artemether-lumefantrine (four-dose regimen) for treating uncomplicated falciparum malaria. Cochrane Database Syst Rev. 2006;19(2):CD005965.

9. Omari AA, Gamble CL, Garner P. Artemether-lumefantrine (six-dose regimen) for treating uncomplicated falciparum malaria. Cochrane Database Syst Rev. 2005;4(CD005564).

10. Travassos, Mark A, Laufer, et al. Resistance to antimalarial drugs: molecular, pharmacologic, and clinical considerations. Pediatr Res. 2009;65(5):64R-70R.

11. White NJ, Van Vugt M, Ezzet F, Ezzet F. Clinical Pharmacokinetics and pharmacodynamics of artemetherlumefantrine. Clin Pharmacokinet. 1999;37:105-125.

12. Kokwaro G, Mwai L, Nzila A. Artemether/lumefantrine in the treatment of uncomplicated falciparum malaria. Expert Opin Pharmacother. 2007;8:75-94.
13. Golenser J, Waknine JH, Krugliak M, et al. Current perspectives on the mechanisms of action of artemisinins. Int J Parasitol. 2006;36: $1427-1441$.

14. Aweeka FT, German PI. Clinical pharmacology of artemisinin-based combination therapies. Clin Pharmacokinet. 2008;47(2):91-102.

15. Abdulla S, Borrmann S, D'Alessandro U, et al. Efficacy and safety of artemetherlumefantrine dispersible tablets compared with crushed commercial tablets in African infants and children with uncomplicated malaria: a randomised, single-blind, multicentre trial. Lancet. 2008;372:1819-1827.

16. Abdulla S, Sagara I. Dispersible formulation of artemether/lumefantrine: specifically developed for infants and young children. Malar J. 2009; 8(1):1186/475-2875-8-S1-S7.

17. Ezzet F, Mull R, Karbwang J. Population pharmacokinetics and therapeutic response of CGP 56697 (artemether + benflumetol) in malaria parasites. Br J Clin Pharmacol. 1998;46:553-561.

18. White N. Preventing antimalarial drug resistance through combinations. Drug Resistance Updates. 1998;1:3-9.

19. Djimdé A, Lefèvre G. Understanding the pharmacokinetics of Coartem ${ }^{\circledR}$. Malar J. 2009;8(1):1186/475-2875-8-S1-S4.

20. Ezzet F, Van Vugt M, Nosten F, et al. Pharmacokinetics and pharmacodynamics of lumefantrine (benflumetol) in acute falciparum malaria. Antimicrob Agents Chemother. 2000;44:697-704.

21. Ashley EA, Stepniewska K, Lindegårdh N. Pharmacokinetic study of artemether-lumefantrine given once daily for the treatment of uncomplicated multidrugresistant falciparum malaria. Trop Med Int Health. 2007;12:201-208.

22. Premji ZG, Abdulla S, Ogutu B, et al. The content of African diets is adequate to achieve optimal efficacy with fixed-dose artemetherlumefantrine: a review of the evidence. Malar J. 2008;7:244.

23. Michael Makanga SK. The clinical efficacy of artemether/lumefantrine (Coartem $^{\circledR}$ ). Malar J. 2009;8(1):1186/475-2875-8-S1-S5.

24. Van Vugt M, Wilairatana P, Gemperli B, et al. Efficacy of six doses of artemether/lumefantrine (benflumetol) in multidrug-resistant Plasmodium falciparum malaria. Am J Trop Med Hyg. 1999;60: 936-942.

25. Van Vugt M, Looareesuwan S, Wilairatana P, et al. Artemether/ lumefantrine for the treatment of multi-drug resistant falciparum malaria. Trans R Soc Trop Med Hyg. 2000;94:545-548.

26. Lefèvre G, Looareesuwan S, Treeprasertsuk S, et al. A clinical and PK trial of six doses of artemether-lumefantrine for multidrug-resistant Plasmodium falciparum malaria in Thailand. Am J Trop Med Hyg. 2001;64(5-6):247-256.

27. Hatz C, Soto J, Nothdurft HD, et al. Treatment of acute uncomplicated falciparum malaria with artemether/lumefantrine in nonimmune populations: a safety, efficacy and PK study. Am J Trop Med Hyg. 2008;78:241-247.

28. Rojanawatsirivej C,Vijaykadga S,Amklad I, et al. Monitoring the therapeutic efficacy of antimalarials against uncomplicated falciparum malaria in Thailand. Southeast Asian J Trop Med Public Health. 2003; 34:536-541.

29. Bukirwa H, Yeka A, Kamya MR, et al. Artemisinin Combination Therapies for Treatment of Uncomplicated Malaria in Uganda. PLos Clin Trials. 2006;1(1):0010007.

30. Dorsey G, Staedke S, Clark TD, et al. Combination therapy for uncomplicated falciparum malaria in Ugandan children. JAMA. 2007;297:2210-2219.

31. Gürkov R, Eshetu T, Miranda IB, et al. Ototoxicity of artemether/ lumefantrine in the treatment of falciparum malaria: a randomized trial. Malar J. 2008;7:179.

32. Kabanywanyi AM, Mwita A, Sumari D, et al. Efficacy and safety of artemisinin-based antimalarial in the treatment of uncomplicated malaria in children in southern Tanzania. Malar J. 2007;6:146.

33. Kamya MR Yeka A, Bukirwa H, et al. Artemether/lumefantrine versus dihydroartemisinin-piperaquine for treatment of malaria: a randomized trial. PLoS Clin Trials. 2007;2:e20. 
34. Mårtensson A, Strömberg J, Sisowath C, et al. Efficacy of artesunate plus amodiaquine versus that of artemether/lumefantrine for the treatment of uncomplicated childhood Plasmodium falciparum malaria in Zanzibar, Tanzania. Clin Infect Dis. 2005;41:1079-1087.

35. Mårtensson A, Ngasala B, Ursing J, et al. Influence of consecutive-day blood sampling on polymerase chain reaction-adjusted parasitological cure rates in an antimalarial-drug trial conducted in Tanzania. $J$ Infect Dis. 2007;195:597-601.

36. Mohamed AO, Eltaib EH, Ahmed OA, et al. The efficacies of artesunatesulfadoxine-pyrimethamine and artemether/lumefantrine in the treatment of uncomplicated, Plasmodium falciparum malaria, in an area of low transmission in central Sudan. Ann Trop Med Parasitol. 2006;100:5-10.

37. Mukhtar EA, Gadalla NB, El-zaki SEG, et al. A comparative study on the efficacy of artesunate plus sulphadoxine/pyrimethamine versus artemether/lumefantrine in eastern Sudan. Malar J. 2007;6:92.

38. Yeka A, Dorsey G, Kamya MR, et al. Artemether/lumefantrine versus dihydroartemisinin-piperaquine for treating uncomplicated malaria: a randomized trial to guide policy in Uganda. PLOS ONE. 2008(3): e2390.

39. Mulenga M, Van geertruyden JP, Mwananyanda L, et al. Safety and efficacy of lumefantrine-artemether $\left(\right.$ Coartem $\left.^{\circledR}\right)$ for the treatment of uncomplicated Plasmodium falciparum malaria in Zambian adults. Malar J. 2006;5:73.

40. Toovey S. Effectiveness of co-artemether in an unsupervised outpatient setting for the treatment of falciparum malaria. Travel Med Infect Dis. 2008;6(1-2):29-31.

41. Adjei GO, Kurtzhals JA, Rodrigues OP, et al. Amodiaquine artesunate vs artemether/lumefantrine for uncomplicated malaria in Ghanaian children: a randomized efficacy and safety trial with one year follow-up. Malar J. 2008;7:127.

42. Faye B, Ndiaye JL, Ndiaye D, et al. Efficacy and tolerability of four antimalarial combinations in the treatment of uncomplicated Plasmodium falciparum malaria in Senegal. Malar J. 2007;6:80.

43. Koram KA, Abuaku B, Duah N, et al. Comparative efficacy of antimalarial drugs including ACTs in the treatment of uncomplicated malaria among children under 5 years in Ghana. Acta Trop. 2005;95: 194-203.

44. Meremikwu M, Alaribe A, Ejemot R, et al. Artemether/lumefantrine versus artesunate plus amodiaquine for treating uncomplicated childhood malaria in Nigeria: randomized controlled trial. Malar J. 2006;5:43.

45. Owusu-Agyei S, Asante KP, Owusu R, et al. An open label, randomised trial of artesunate + amodiaquine, artesunate + chlorproguanil-dapsone and artemether/lumefantrine for the treatment of uncomplicated malaria. PLoS ONE. 2008;3:e2530.

46. Sagara I, Dicko A, Djimde A, et al. A randomized trial of artesunatesulfamethoxypyrazinepyrimethamine versus artemether/lumefantrine for the treatment of uncomplicated Plasmodium falciparum malaria in Mali. Am J Trop Med Hyg. 2006;75:630-636.

47. Sowunmi A, Gbotosho GO, Happi CT, et al. Therapeutic efficacy and effects of artemether/lumefantrine and amodiaquinesulfalenepyrimethamine on gametocyte carriage in children with uncomplicated Plasmodium falciparum malaria in southwestern Nigeria. Am J Trop Med Hyg. 2007;77:235-241.

48. Sutherland CJ, Ord R, Dunyo S, et al. Reduction of malaria transmission to Anopheles mosquitoes with a sixdose regimen of co-artemether. PLoS Med. 2005;2:e92.

49. Zongo I, Dorsey G, Rouamba N, et al. Artemether/lumefantrine versus amodiaquine plus sulfadoxine-pyrimethamine for uncomplicated falciparum malaria in Burkina Faso: a randomized non-inferiority trial. Lancet. 2007;369:491-498.

50. Zongo I, Dorsey G, Rouamba N, et al. Randomized comparison of amodiaquine plus sulfadoxine-pyrimethamine, artemether/lumefantrine, and dihydroartemisinin-piperaquine for the treatment of uncomplicated Plasmodium falciparum malaria in Burkina Faso. Clin Infect Dis. 2007;45:1453-1461.
51. Fanello CI, Karema C, van Doren W, et al. A randomised trial to assess the safety and efficacy of artemether/lumefantrine $\left(\right.$ Coartem $\left.{ }^{\circledR}\right)$ for the treatment of uncomplicated Plasmodium falciparum malaria in Rwanda. Trans R Soc Trop Med Hyg. 2007;101:344-350.

52. Guthmann JP Cohuet S, Rigutto C, et al. High efficacy of two artemisinin-based combinations (artesunate + amodiaquine and artemether + lumefantrine) in Caala, Central Angola. Am J Trop Med Hyg. 2006;75:143-145.

53. Ndayiragije A, Niyungeko D, Karenzo J, et al. Efficacité de combinaisons thérapeutiques avec des dérivés de l'artémisinine dans le traitement de l'accès palustre noncomplique au Burundi. Trop Med Int Health. 2004;9:673-679.

54. Van den Broek I, Kitz C, Al Attas S, et al. Efficacy of three artemisinin combination therapies for the treatment of uncomplicated Plasmodium falciparum malaria in the Republic of Congo. Malar J. 2006;5:113.

55. Krudsood S, Chalermrut K, Pengruksa C, et al. Comparative clinical trial of two-fixed combinations dihydroartemisinin-napthoquinetrimethoprim (DNP ${ }^{\circledR}$ ) and artemether/lumefantrine Coartem ${ }^{\circledR} /$ Riamet $^{\circledR}$ ) in the treatment of acute uncomplicated falciparum malaria in Thailand. Southeast Asian J Trop Med Public Health. 2003;34: 316-321.

56. Stohrer JM, Dittrich S, Thongpaseuth V, et al. Therapeutic efficacy of artemether/lumefantrine and artesunate-mefloquine for treatment of uncomplicated Plasmodium falciparum malaria in Luang Namtha Province, Lao People's Democratic Republic. Trop Med Int Health. 2004;9:1175-1183.

57. Mayxay M, Khanthavong M, Lindegårdh N, et al. Randomized comparison of chloroquine plus sulfadoxine-pyrimethamine versus artesunate plus mefloquine versus artemether/lumefantrine in the treatment of uncomplicated falciparum malaria in the Lao People's Democratic Republic. Clin Infect Dis. 2004;39:1139-1147.

58. Ratcliff A, Siswantoro H, Kenangalem E, et al. Two fixed-dose artemisinin combinations for drug-resistant falciparum and vivax malaria in Papua, Indonesia: an open-label randomised comparison. Lancet. 2007;369:757-765.

59. Hutagalung R, Paiphun L, Ashley EA, et al. A randomized trial of artemether/lumefantrine versus mefloquine-artesunate for the treatment of uncomplicated multi-drug resistant Plasmodium falciparum on the western border of Thailand. Malar J. 2005;4:46.

60. Van den Broek IV, Maung UA, Peters A, et al. Efficacy of chloroquine + sulfadoxine-pyrimethamine, mefloquine + artesunate and artemether + lumefantrine combination therapies to treat Plasmodium falciparum malaria in the Chittagong Hill Tracts, Bangladesh. Trans $R$ Soc Trop Med Hyg. 2005;99:727-735.

61. Haque R, Thriemer K, Wang Z, et al. Therapeutic efficacy of artemether/ lumefantrine for the treatment of uncomplicated Plasmodium falciparum malaria in Bangladesh. Am J Trop Med Hyg. 2007;76:39-41.

62. Thapa S, Hollander J, Linehan M, et al. Comparison of artemether/ lumefantrine with sulfadoxine-pyrimethamine for the treatment of uncomplicated falciparum malaria in Eastern Nepal. Am J Trop Med Hyg. 2007;77:423-430.

63. Jansen FH, Lesaffre E, Penali LK, et al. Assessment of the relative advantage of various artesunate-based combination therapies by a multi-treatment Bayesian random-effects meta-analysis. Am J Trop Med Hyg. 2007(77):1005-1009.

64. Piola P, Fogg C, Bajunirwe F, et al. Supervised versus unsupervised intake of six-dose artemether-lumefantrine for treatment of acute, uncomplicated Plasmodium falciparum malaria in Mbarara, Uganda: a randomised trial. Lancet. 2005;365(9469):1467-1473.

65. Achan J, Tibenderana J, Kyabayinze D, et al. Effectiveness of quinine versus artemether-lumefantrine for treating uncomplicated falciparum malaria in Ugandan children: randomised trial. BMJ. 2009;339(2763):2763.

66. Falade C, Makanga M, Premji Z, et al. Efficacy and safety of artemether/ lumefantrine $\left(\right.$ Coartem $\left.^{\circledR}\right)$ tablets (six-dose regimen) in African infants and children with acute, uncomplicated falciparum malaria. Trans $R$ Soc Trop Med Hyg. 2005(99):459-467. 
67. Falade C, Manyando C. Safety profile of Coartem ${ }^{\circledR}$ : the evidence base. Malar J. 2009;8 Suppl 1:S6.

68. Toovey S, Jamieson A. Audiometric changes associated with the treatment of uncomplicated falciparum malaria with coartemether. Trans R Soc Trop Med Hyg. 2004;98:261-267.

69. Miller LG, Panosian CB. Ataxia and slurred speech after artesunate treatment for falciparum malaria. N Engl J Med. 1997;336:1328.

70. Cousin M, Kummerer S, Lefèvre G, et al. Advisory Committee Briefing Book. Coartem ${ }^{\circledR}$ (artemether/lumefantrine) tablets for the treatment of malaria in patients with acute, uncomplicated infections due to Plasmodium falciparum or mixed infections including $P$. falciparum. http://wwwfdagov/ohrms/dockets/ac/08/briefing/2008-4388b1-02Novartispdf. 2008

71. Bakshi R, Hermeling-Fritz I, Gathmann I, et al. An integrated assessment of the clinical safety of artemether-lumefantrine:a new oral fixed-dose combination antimalarial drug. Trans R Soc Trop Med Hyg. 2000;94:419-424.

72. Nankabirwa Zurovac D, Njogu JN, et al. Malaria misdiagnosis in Uganda -implications for policy change. Malar J. 2009;16(8):66.

73. Talisuna AO, Staedke SG, D'Alessandro U. Pharmacovigilance of antimalarial treatment in Africa: is it possible? Malar J. 2006;5:50.

74. Barnes KI, Little F, Smith PJ, et al. Sulfadoxine-pyrimethamine PKs in malaria: pediatric dosing implications. Clin Pharmacol Ther. 2006; 80:582-596.

75. Price RN, Hasugian AR, Ratcliff A, et al. Clinical and pharmacological determinants of the therapeutic response to dihydroartemisininpiperaquine for drug-resistant malaria. Antimicrob Agents Chemother. 2007;51:4090-4097.

76. ter Kuile FO, Terlouw DJ, Kariuki SK, et al. Impact of permethrin-treated bed nets on malaria, anemia, and growth in infants in an area of intense perennial malaria transmission in western Kenya. Am J Trop Med Hyg. 2003;68:68-77.

77. Slutsker L, Taylor TE, Wirima JJ, et al. In-hospital morbidity and mortality due to malaria-associated severe anaemia in two areas of Malawi with different patterns of malaria infection. Trans $R$ Soc Trop Med Hyg. 1994;88:548-551.

78. Schellenberg D, Menendez C, Kahigwa E, et al. African children with malaria in an area of intense Plasmodium falciparum transmission: features on admission to the hospital and risk factors for death. Am J Trop Med Hyg. 1999;61:431-438.

79. Nosten F, White NJ. Artemisinin-based combination treatment of Falciparum malaria. Am J Trop Med Hyg. 2007;77(6):181-192.

80. Longo M, Zanoncelli S, Manera D, et al. Effects of the antimalarial drug dihydroartemisinin (DHA) on rat embryos in vitro. Reprod Toxicol. 2006;21(1):83-93.

81. Dellicour S, O ter Kuile F, Stergachis A. Pregnancy exposure registries for assessing antimalarial drug safety in pregnancy in malaria-endemic countries. PLoS Med. 2008;5(9):187.

82. Nosten F, McGready R, Mutabingwa T. Case management of malaria in pregnancy. Lancet Infect Dis. 2007;7(2):118-125.

83. McGready R, Cho T, Keo NK, et al. Artemisinin antimalarials in pregnancy: a prospective treatment study of 539 episodes of multidrugresistant Plasmodium falciparum. Clin Infect Dis. 2001;33(12):2009-2016.

84. Dellicour S, Hall S, Chandramohan D. The safety of artemisinins during pregnancy: a pressing question. Malar J. 2007;14(6):15.

85. Adam I, Elhassan EM, Omer EM, Abdulla MA. Safety of artemisinins during early pregnancy, assessed in 62 Sudanese women. Ann Trop Med Parasitol. 2009;103(3):205-210.

86. Manyando C, Mkandawire R, Puma L, et al. Safety profile artemetherlumefantrine (AL; Coartem ${ }^{\circledR}$ ) compared sulfadoxine-pyrimethamine (SP) pregnant women symptomatic malaria: preliminary results of an observational study. Abstract 570, 57th Meeting of the American Society of Tropical Medicine and Hygiene (ASTMH), New Orleans, Louisiana, USA, December 2008.

87. Tarning J, McGready R, Lindegardh N, et al. Population PKs of lumefantrine in pregnant women treated with artemether-lumefantrine for uncomplicated P. falciparum malaria. Antimicrob Agents Chemother. 2009;53:3837-3846.
88. McGready R, Stepniewska K, Lindegardh N, et al. The pharmacokinetics of artemether and lumefantrine in pregnant women with uncomplicated falciparum malaria. Eur J Clin Pharmacol. 2006;62(12): 1021-1031.

89. McGready R, Tan SO, Ashley EA, et al. A randomised controlled trial of artemether-lumefantrine versus artesunate for uncomplicated Plasmodium falciparum treatment in pregnancy. PLoS Med. 2008;23(5):e253.

90. Whitworth J, Morgan D, Quigley M, et al. Effect of HIV-1 and increasing immunosuppression on malaria parasitaemia and clinical episodes in adults in rural Uganda: a cohort study. Lancet. 2000;356(9235): 1051-1056.

91. French N, Nakiyingi J, Lugada E, et al. Increasing rates of malarial fever with deteriorating immune status in HIV-1-infected Ugandan adults. AIDS. 2001;15(7):899-906.

92. Francesconi P, Fabiani M, Dente MG, et al. HIV, malaria parasites, and acute febrile episodes in Ugandan adults: a case-control study. AIDS. 2001;15(18):2445-2450.

93. Atzori C, Bruno A, Chichino G, et al. HIV-1 and parasitic infections in rural Tanzania. Ann Trop Med Parasitol. 1993;87(6):585-593.

94. Van Eijk AM, Ayisi JG, Ter Kuile FO, et al. HIV increases the risk of malaria in women of all gravidities in Kisumu, Kenya. AIDS. 2003;17(4):595-603.

95. Verhoeff $\mathrm{FH}$, Brabin BJ, Hart CA, et al. Increased prevalence of malaria in HIV-infected pregnant women and its implications for malaria control. Trop Med Int Health. 1999;4(1):5-12.

96. Steketee RW, Wirima JJ, Slutsker L, et al. Comparability of treatment groups and risk factors for parasitemia at the first antenatal clinic visit in a study of malaria treatment and prevention in pregnancy in rural Malawi. Am J Trop Med Hyg. 1996;55(1):17-23.

97. Shulman. Importance and prevention of malaria in pregnancy. Trans R Soc Trop Med Hyg. 2003;97(1):30-35.

98. Bloland PB, Wirima JJSR, Chilima B, et al. Maternal HIV infection and infant mortality in Malawi: evidence for increased mortality due to placental malaria infection. AIDS. 1995;9(7):721-726.

99. Byakika-Kibwika P, Ddumba E, Kamya M. Effect of HIV-1 infection on malaria treatment outcome in Ugandan patients. African Health Sciences. 2007;7(2):86-92.

100. Kamya MR, Kigonya CN, McFarland W. HIV infection may adversely affect clinical response to chloroquine therapy for uncomplicated malaria in children. AIDS. 2001;15(9):1187-1188.

101. Kamya MR, Gasasira AF, Yeka A, et al. Effect of HIV-1 infection on antimalarial treatment outcomes in Uganda: a population-based study. J Infect Dis. 2006;193(1):9-15.

102. Colebunders R, Bahwe Y, Nekwei W, et al. Incidence of malaria and efficacy of oral quinine in patients recently infected with human immunodeficiency virus in Kinshasa, Zaire. J Infect Dis. 1990;21(2):167-173.

103. Van Geertruyden JP, Mulenga M, Mwananyanda L, et al. HIV-1 immune suppression and antimalarial treatment outcome in Zambian adults with uncomplicated malaria. $J$ Infect Dis. 2006;194(7): 917-925.

104. Birku YME, Björkman A, Wolday D. Delayed clearance of Plasmodium falciparum in patients with human immunodeficiency virus co-infection treated with artemisinin. Ethiop Med J. 2002;1:17-26.

105. Patnaik P, Jere CS, Miller WC, et al. Effects of HIV-1 serostatus, HIV-1RNA concentration, and CD4 cell count on the incidence of malaria infection in a cohort of adults in rural Malawi. J Infect Dis. 2005;192:984-991.

106. Van Geertruyden JP, Mulenga M, Kasongo W, et al. CD4 T-cell count and HIV-1 infection in adults with uncomplicated malaria. $J$ Acquir Immune Defic Syndr. 2006;43(3):363-367.

107. Khoo S, Back D, Winstanley P. The potential for interactions between antimalarial and antiretroviral drugs. AIDS. 2005;19(10):995-1005.

108. Moses R. Kamya, Adoke Yeka, Hasfa Bukirwa, et al. Artemetherlumefantrine versus dihydroartemisinin-piperaquine for treatment of malaria: a randomized trial. PLoS Clin Trials. 2007;2(5):1371. 
109. Gasasira AF, Kamya MR, Achan J, et al. High risk of neutropenia in HIV-infected children follwoing treatment with artesunate for uncomplicated malaria in Uganda. CID. 2008;46:985-991.

110. German P, Parikh S, Lawrence J, et al. Lopinavir/ritonavir affects PK exposure of artemether/lumefantrine in HIV-uninfected healthy volunteers. J Acquir Immune Defic Syndr. 2009;51(4):424-429.

111. Sousa M, Pozniak A, Boffito M. Pharmacokinetics and pharmacodynamics of drug interactions involving rifampicin, rifabutin and antimalarial drugs. J Antimicrob Chemother. 2008;62(5):872-878.

112. Lefevre G. PK and electrocardiographic pharmacodynamics of artemether-lumefantrine $\left(\right.$ Riame $\left.^{\mathbb{R}}\right)$ with concomitant administration of ketoconazole in healthy subjects. Br J Clin Pharmacol. 2002;54:485-492.

113. Bloland PB. Drug resistance in malaria. WHO/CDS/CSR/DRS/20014. 2001.

114. Huong NM, Hweitt S, Davis TM. Resistance of P. falciparum to antimalarial drugs in a highly endemic area in southern Viet Nam: a study in vivo and vitro. Trans R Soc Trop Med Hyg. 2001;95:325-329.

115. Yang H, Liu D, Yang Y. Changes in susceptibility of Plasmodium falciparum to artesunate in vitro in Yunnan Province, China. Trans $R$ Soc Trop Med Hyg. 2003;97:226-228.

116. Gordon P, Kelly R, Hedderwick S. Failure of artemether-lumefantrine (Riamet) to clear Plasmodium falciparum in non immune traveller. Abstract. First Interanational Conference of Travel Medicine and Infectious Disease, London, UK. 2005.
117. Yangsutaka Mizuno, Yasuyuki Kato, Koichiro Kudo, et al. First case of Treatment failure of artemether-lumefantrine in a Japanese traveler with imported falciparum malaria. Jpn J Infect Dis. 2009; 62:139-141.

118. Sisowath C, Stromberg J, Martensson A, et al. In vivo selection of Plasmodium falciparum pfmdr1 $86 \mathrm{~N}$ coding alleles by artemetherlumefantrine (Coartem). J Infect Dis. 2005;191:1014-1017.

119. Duraisingh MT, Jones P, Sambou I, von Seidlein L, Pinder M, Warhurst DC. The tyrosine-86 allele of the pfmdr1 gene of Plasmodium falciparum is associated with increased sensitivity to the anti-malarials mefloquine and artemisinin. Mol Biochem Parasitol. 2000;108: 13-23.

120. Newton PN, Fernandez FM, Plancon A, et al. A collaborative epidemiological investigation into the criminal fake artesunate trade in South East Asia. PLoS Med. 2008;5:e32.

121. Atemnkeng MA, De Cock K, Plaizier-Vercammen J. Quality control of active ingredients in artemisinin-derivative antimalarials within Kenya and DR Congo. Trop Med Int Health. 2007;12:68-74.

122. Bate R, Coticelli P, Tren R. Antimalarial drug quality in the most severely malarious parts of Africa-a six country study. PLOS ONE. 2008;3:e2132.
Therapeutics and Clinical Risk Management

\section{Publish your work in this journal}

Therapeutics and Clinical Risk Management is an international, peerreviewed journal of clinical therapeutics and risk management, focusing on concise rapid reporting of clinical studies in all therapeutic areas, outcomes, safety, and programs for the effective, safe, and sustained use of medicines. This journal is indexed on PubMed Central, CAS,

\section{Dovepress}

EMBase, Scopus and the Elsevier Bibliographic databases. The manuscript management system is completely online and includes a very quick and fair peer-review system, which is all easy to use. Visit $\mathrm{http}: / /$ www.dovepress.com/testimonials.php to read real quotes from published authors. 